\title{
Note sur certaines manifestations du réveil religieux de 1840 dans la paroisse Notre-Dame de Québec
}

\section{René Hardy}

Volume 35, 1968

URI : https://id.erudit.org/iderudit/1007309ar

DOI : https://doi.org/10.7202/1007309ar

Aller au sommaire du numéro

Éditeur(s)

Les Éditions Historia Ecclesiæ Catholicæ Canadensis Inc.

ISSN

0318-6172 (imprimé)

1927-7067 (numérique)

Découvrir la revue

Citer cette note

Hardy, R. (1968). Note sur certaines manifestations du réveil religieux de 1840 dans la paroisse Notre-Dame de Québec. Sessions d'étude - Société canadienne d'histoire de l'Église catholique, 35, 81-98. https://doi.org/10.7202/1007309ar

Tous droits réservés ㄷ Les Éditions Historia Ecclesiæ Catholicæ Canadensis Inc., 1968
Ce document est protégé par la loi sur le droit d'auteur. L'utilisation des services d'Érudit (y compris la reproduction) est assujettie à sa politique d'utilisation que vous pouvez consulter en ligne.

https://apropos.erudit.org/fr/usagers/politique-dutilisation/ 


\section{Note sur certaines manifestations du réveil religieux de 1840 dans la paroisse Notre-Dame de Québec}

Lors d'une conférence prononcée en $1942^{1}$, l'historien Groulx fait une esquisse très peu élogieuse de la piété populaire durant la première moitié du $\mathrm{XIX}^{\circ}$ siècle. Il situe aux alentours de 1840 , à l'occasion des retraites prêchées par Mgr de Forbin-Janson, l'amorce d'un mouvement appelé réveil religieux. En fait, les décennies 1840 . 1860 marquent une étape décisive dans la réorganisation des structures ecclésiastiques et dans la cristallisation de la pensée religieuse québécoise. C'est l'époque de l'émigration des clercs et religieux français (Oblats en 1841, Jésuites en 1842, Clercs Saint-Viateur et Pères de Sainte-Croix en 1847, Frères des Écoles Chrétiennes en 1837, Souurs du Sacré-Cour en 1842, Sœurs du Bon-Pasteur d'Angers en 1844, Scurs de Sainte-Croix en 1847, Sœurs de la Présentation en 1853, Sœurs de Jésus-Marie en $1855^{2}$ ), de la nomination du premier évêque de Montréal et de l'archevêque de Québec et surtout des débuts de l'ultramontanisme au Bas-Canada. L'effectif ecclésiastique monte en flèche durant cette période. Louis-Edmond Hamelin a démontré que dans les années $1830,1840,1850$ et 1860 , il y avait une moyenne de $1834,1185,1080$ et 893 fidèles par prêtre et que le nombre des prêtres passaient respectivement selon les dates de 225 à 464,620 et $948^{3}$. A la paroisse Notre-Dame de Québec, cinq prêtres en 1840 , y compris le vicaire de l'église St. Patrick, desservent environ 16,560 paroissiens. Trois ans plus tard, un autre vicaire s'ajoute au groupe; cette situation dure jusqu'à l'ouverture de l'église Saint-Jean-Baptiste,

1 Lionel Groulx, e La situation religieuse au Canada français vers 1840 ,, S.C.H.E.C., 1941-1942, pp. 51-75.

2 Gérard Filteau, Organisation scolaire de la province de Québec, Montréal, Centre de Psychologie et de Pédagogie, 1954, pp. 66 et 124.

De nombreuses autres congrégations religieuses ont été fondées au Québec. Ce sont les Sœurs des Saints Noms de Jésus et de Marie en 1843, les Sœurs de la Charité de la Providence en 1843, les Sœurs Grises de la Croix en 1845, les Sœurs de Sainte-Anne en 1848, les Sœurs Grises de la Charité de Québec en 1849, les Sœurs du Bon-Pasteur de Québec en 1850 et les Sœurs de l'Assomption de la Sainte-Vierge en 1853.

3 Louis-Edmond Hamelin, - Evolution numérique séculaire du clergé catholique dans le Québec ,, Recherches Sociographiques, vol. 2, avril-juin 1961, pp. 237-238. 
en 1848, alors que le curé se voit obligé de nommer un desserrant pour la future paroisse. L'effectif ecclésiastique diminue sensiblement en 1855 : on ne compte plus que trois prêtres ${ }^{4}$. Mais, selon le vicaire Henri-Raymond Casgrain, leurs occupations sont peu absorbantes ${ }^{5}$. Cette situation peut s'expliquer par le fait qu'il n'y a pas eu de malheurs collectifs ${ }^{6}$ dans la paroisse depuis le dernier choléra de 1854 et que la misère sociale est en partie soulagée par les organismes et les institutions religieuses. Parmi les institutions existantes, il y a le Séminaire de Québec, le couvent des Ursulines, l'Hôtel-Dieu et l'Hôpital Général, auxquelles s'ajoutent durant la période étudiée, cinq nouvelles corporations religieuses dans la seule paroisse Notre-Dame. Ce sont les Frères des Écoles Chrétiennes en 1843, l'Hospice des Sœurs de la Charité en 1848, l'Asile du Bon-Pasteur en 1850, l'Hospice SaintJoseph-de-la-Maternité en 1852 et l'asile Sainte-Brigitte ${ }^{7}$. Chacune de ces institutions, en plus des organismes laïques tels que les sociétés Saint-Vincent-de-Paul, de l'Éducation, des Bons Livres et des Dames Charitables ${ }^{8}$, devient, soit pour des ouvres strictement religieuses, soit pour des œuvres sociales, un moyen d'action à la disposition des curés.

C'est donc dans cette période de diversification des institutions ecclésiastiques que nous avons voulu étudier le rôle du curé et l'empire de la religion sur les paroissiens de Notre-Dame de Québec. Nos préoccupations méthodologiques ont fait immédiatement ressortir les difficultés de qualifier et de quantifier des sentiments et des comportements. Pour en arriver à une approximation valable de la situation, il faut nécessairement se référer aux principales manifestations religieuses de la période antérieure et comparer, statistiques à l'appui, les activités des nombreuses confréries religieuses, ce qui est impossible vu l'absence de monographies en ces domaines. En conséquence, que le lecteur ne cherche pas dans cet écrit une évaluation, précise à l'échelle paroissiale, de ce que les historiens ont appelé le réveil religieux de 1840 ! Il est tout au plus une vérification de son existence.

Nous nous sommes restreint, dans une première partie, à décrire les retraites, les indulgences, les processions et les offices, tout en tenant compte de quelques renseignements et données statistiques relatifs

4 Nous ne comptons plus ici les desservants de l'église St. Patrick et Saint-Jean-Baptiste.

5 H.-R. Casgrain, Souvenances Canadiennes, tome III, p. 32.

6 Voici la liste des malheurs collectifs qui ont atteint les citoyens de Québec entre 1830 et 1854 : maladies contagieuses (choléra, typhus, etc.) en $1832,1834,1847,1849,1851,1852$ et 1854; incendie de la Basse-Ville en 1836, du quartier Saint-Roch en mai 1845, du quartier Saint-Jean en juin 1845 et du théâtre Saint-Louis en 1846.

7 Le Courrier du Canada, 21 octobre 1870.

8 Nous pourrions ajouter les confréries du Saint-Scapulaire, de la SainteFamille. de Grinte-Anne. dec congréganistes et ausi les aconciations de la SainteEnfance et du Denier de Saint-Pierre. 
à la période précédente. Ensuite, nous avons émis quelques hypothèses en vue de l'explication de cette ferveur, afin de pouvoir apprécier la profondeur des sentiments manifestés.

\section{Forbin-Janson et les retraites au Bas-Canada}

Le réveil religieux caractéristique de la période s'est d'abord manifesté par la retraite prêchée à l'automne 1840, par monseigneur de Forbin-Janson, évêque de Nancy et de Toul, dont le voyage en Amérique n'est qu'une péripétie d'une vie très mouvementée. Fils de famille noble, Charles de Forbin-Janson suit sa mère en exil au temps de la Révolution. Rentré à Paris sous l'Empire, il se retire du Conseil d'État et refuse la mission diplomatique que lui offre Napoléon pour y préférer les études ecclésiastiques au séminaire de Saint-Sulpice. Il rencontre là Eugène de Mazenod avec qui il se lie d'amitié. Ensemble, ils se découvrent des aptitudes pour la vie de missionnaire. Mais à l'encontre de celui qui deviendra le fondateur des Oblats de MarieImmaculée, Forbin-Janson désire alors évangéliser la Chine. Des circonstances l'en détournent. Le 9 janvier 1815 il met sur pied l' "Euvre des Missions pour l'intérieur de la France ${ }^{9}$ » et amorce ainsi une carrière de prédicateur qui ne cessera d'étonner la France. D'abord parce qu'ultramontain et légitimiste farouche, il ne peut s'empêcher, dans ses sermons, d'associer religion et politique : c'est souvent au cri de "vive le roi " que la foule élève la croix de mission à la fin de ses prédications. Mais aussi parce que Forbin-Janson n'est pas avare de moyens pour toucher la sensibilité de son auditoire. A Marseille, en 1820 , il prêche dans un cimetière, sur une fosse ouverte, " en présence des outils du fossoyeur et des ossements qu'on avait pu recueillir ${ }^{10}$. Ailleurs, ses disciples prêchent devant un catafalque illuminé par des bougies placées à l'intérieur de citrouilles sculptées en forme de tête de mort ${ }^{11}$. Curieux et dévots accourent. Certains le suivent dans ses déplacements, avides d'entendre cet habile orateur. Mais s'il attire les foules, il n'est pas moins détesté par les libéraux et les anticléricaux qui ne lui pardonnent pas ses allusions politiques et ses excès de parole.

En 1824, il est sacré évêque de Nancy et de Toul. Ses ennemis politiques et religieux qui, pour un temps, auraient pu l'oublier, sont bien vite provoqués par des mandements où la dynastie des Bourbons est considérée comme la seule acceptable par "le(s) véritable(s) disciple(s) du Christ ${ }^{12}$ ». Quand la révolution éclate, en juillet 1830 ,

9 Paul Lesourd, Un grand Cour Missionnaire, Monseigneur de ForbinJanson, 1785-1844, Paris, Flammarion, 1944, p. 26.

10 Ibid., p. 41.

11 Ibid., p. 41.

12 Ibid., p. 48. 
Forbin-Janson s'exile, poursuivi et menacé par ceux-là qui, depuis quinze ans, contiennent leur colère. Et en vain, jusqu'à sa mort, réclamera-t-il de Louis-Philippe la permission de regagner son diocèse. Toutefois, cet exil prolongé, loin de mettre fin à sa carrière apostolique, le conduit en Amérique, particulièrement au Bas-Canada où en 1841 et 1842 , il prêche de nombreuses retraites populaires.

Alors que sous l'Empire, mais davantage sous la Restauration, les catholiques français voient les missionnaires narcourir la France et prêcher les « missions populaires " en vue de lutter contre la déchristianisation des masses, les Québécois de la génération 1840, malgré les relents anticléricaux des insurrections, ignorent encore cette forme de prédication ${ }^{13}$. Et sur ce point, les retraites de Forbin-Janson semblent être les premières depuis la Conquête ${ }^{14}$. Quant aux retraites pastorales, les innovations québécoises suivent de près le renouveau clérical français. Abolies par la Révolution, elles sont timidement reprises en France, en 1818, "mais c'est seulement à partir de 1832, semble-t-il, que le mouvement s'accentue et que cette pratique s'introduit graduellement dans tous les diocèse ${ }^{15}$ \%. A Montréal, le clergé assiste à sa première retraite en 1839. Elle est prêchée par un jésuite français du Kentucky, le Père Pierre Chazelle. L'année suivante, le grand vicaire Alexis Mailloux convoque quelques membres du clergé à Sainte-Anne-de-laPocatière pour la première retraite pastorale de la région de Québec ${ }^{16}$. En 1841, Mgr Signay systématise cette œuvre d'éducation cléricale en réunissant 102 prêtres, pendant huit jours, sous les auspices de $\mathrm{Mgr}$ de Forbin-Janson.

\section{Les retraites à Notre-Dame de Québec}

Arrivé à New-York le 19 octobre 1839, l'évêque de Nancy prêche quelques jours avant de repartir pour la Nouvelle-Orléans où, selon ses mots, "il donne une mission qui dure tout le carême 1840 ", et " réussit à ranimer une foi presque éteinte dans (sic) une population

1. Gasten CArrì̀re, Histoire Documentaire de la Congrégation des O.M.I., Ottawa, L'niversité d'Ottawa, 1959, p. 40.

L'auteur écrit que la prédication des retraites se faisait au $\mathrm{XVII}^{e}$ siècle.

14 Des retraites paroissiales avaient été prêchées, quelques mois avant celles de Forbin-Janson, à Sainte-Anne, Saint-Roch, Saint-Pascal et Saint-André, mais l'arrivée de l'évêque de Nancy en établit la coutume dans presque toutes les paroisses du Bas-Canada. A ce sujet, consulter le B.R.H., $\mathbf{n}^{\circ}$ 7, 1901, pp. 275-276.

15 Robert Sylvain, La vie et l'cuvre de Henry de Courcy, (1820-1861), Québec, Presses Universitaires Laval, 1955, p. 68.

16 Treize prêtres seulement assistaient à cette retraite. Elle avait été demandée à l'évêque par les abbés Alexis Mailloux et Joseph Aubry. Si nous nous référons aux termes de la demande faite à l'évêque, nous conviendrons qu'elle n'est pas une retraite générale du clergé : * Votre Grandeur aurait-elle objection à ce que les prêtres qui ont fait la retraite des paroisses (voir note 2 p. 7) se réunissent à Sainte-Anne pour y faire une retraite de cinq jours? Si quelques autres curés voulaient se réunir à nous, votre Grandeur le leur permettrait-elle? . B.R.H., $\mathrm{n}^{\circ}$ 7, 1901, p. 276. 
de près de soixante mille catholiques, qui n'avaient que trois prêtres pour les desservir, et qui n'avaient pas le courage de réparer trois pauvres églises qui tombaient en ruine ${ }^{17}$ ». Sa réputation d'illustre prédicateur ne tarde pas à se propager à travers toute l'Amérique catholique : l'évêque de Montréal en prend connaissance et l'invite à prêcher la retraite de ses curés, qui doit commencer le 28 août. Mais des contretemps retardent son arrivée au Canada jusqu'au 4 septembre, date à laquelle il se présente à Québec et manifeste le désir de prêcher " une mission " à la cathédrale. Il ne peut réaliser son désir que sur les instances du curé Baillargeon, car l'évêque de Québec jugeait le temps inopportun pour réunir les fidèles ${ }^{18}$.

La retraite débute le 13 septembre; elle doit durer une semaine et réunir tous les catholiques de la ville, y compris ceux de la paroisse Saint-Roch. La foule est si empressée qu'on doit continuer la prédication jusqu'au 27 septembre :

Il serait difficile, écrit le curé, de peindre le zèle et l'empressement des citoyens de Québec pour assister aux sermons durant toute la retraite. Le matin, l'église était remplie autant qu'elle pouvait l'être de femmes; et le soir elle avait peine à contenir tous les hommes. C'était un spectacle édifiant de voir tant de gens fatigués des travaux de la journée, accourir de toutes les parties de la ville, et demeurer attentifs à la parole de Dieu durant une heure et demie, et quelquefois deux heures que duraient les sermons - debout, baignés de sueur, au milieu d'une chaleur étouffante qui leur permettrait à peine de respirer 19 .

Voilà une description intéressante de l'esprit qui anime l'auditoire de l'évêque de Nancy. Mais peut-on imaginer que la prédication de la parole de Dieu soit devenue subitement si intéressante ? L'atmosphère qui entoure ces sermons et les talents de l'orateur doivent, plus que l'essence des discours, contribuer à leur succès. Nous en conviendrons à la lecture de la description qu'en fait le Canadien du 20 janvier 1841 :

... Comme il renverse la pierre des tombeaux ! Comme il fait sortir vivants les squelettes poudreux des demeures sépulcrales! Et lorsque, à sa parole, la mort a pesé sur l'abîme, lorsque l'abîme s'est dilaté, puis refermé, comme il appelle l'éternité ! Et l'éternité semble accourir à sa voix : c'est alors que, s'élevant sur son auditoire, l'œil étincelant, la voix sourde, il déroule devant lui les horreurs des gouffres infernaux, qu'il rend présents à tous les esprits, et comme ouverts sous cette immense assemblée. Entendez les accents terribles de sa voix qu'il fait courir comme des roulements de tonnerre sous les arches du temple. Ajoutez à cela que c'est au milieu de la nuit, au reflet de quelques pâles

17 C.P., 27 septembre 1840. Mgr de Nancy a tenu ces propos au curé Baillargeon qui les a transcrits textuellement dans ses Cahiers des Prônes, tel qu'il l'indique à la fin de son texte.

18 Ibid.

19 C.P., 27 septembre 1840. 
flambeaux, qu'il fait entendre ces paroles d'épouvante et qu'il ouvre les sombres cavernes et les désolations de l'éternité. C'est avec raison qu'il dit n'avoir pas voulu effrayer l'esprit timide des mères et des épouses par l'appareil épouvantable de la dernière catastrophe 20 .

Il prêche en tout 24 sermons précédés et suivis du chant des cantiques qui à son dire, « disposent merveilleusement les cœurs à profiter des sermons " et opèrent parfois chez les pécheurs des conversions étonnantes, comme il a pu le constater lors de ses nombreuses retraites ${ }^{21}$. Cette pratique, renouvelée lors de la retraite de 1843, entre graduellement dans la tradition religieuse québécoise. Toutefois, le curé prend soin d'avertir ses paroissiens qu'elle n'est pas nouvelle et que déjà elle se pratiquait dans l'Église primitive ${ }^{22}$.

Bref, la prédication de Forbin-Janson semble être l'exploitation maximum du premier moyen à la disposition du clergé pour raviver la ferveur religieuse des Québécois. Les termes employés par le curé Baillargeon pour annoncer la retraite ne laissent pas de doutes à ce sujet :

... Nous nous contentons de vous conjurer au nom du Seigneur qui va vous faire entendre sa voix avec tant d'éclat, et vous presser avec tant d'insistance par la bouche de son envoyé, de ne point endurcir vos consciences, de profiter de ce temps favorable, de ces jours de salut, et de ne pas recevoir en vain la grâce de Dieu. Eloignez-vous des compagnies; évitez la dissipation, vivez dans le recueillement, rentrez en vous-même, sondez bien vos consciences, voyez en quel état elles sont devant Dieu, gémissez sur vos péchés, pratiquez la mortification et priez beaucoup ${ }^{23}$.

Les résultats ne se font pas attendre: les confessionnaux ne suffisent pas à recevoir les 10,000 femmes et hommes ${ }^{24}$ qui assistent quotidiennement aux sermons du matin et du soir. Les prêtres du séminaire et des paroisses environnantes, en tout 35 confesseurs, ne peuvent en une semaine entendre toutes les confessions. \& On estime à plus de mille le nombre des personnes éloignées depuis plusieurs années de la confession, qui s'approchèrent des sacrements durant la retraite ${ }^{25}$."

Cette première retraite produit des résultats inespérés. Elle consacra d'abord les retraites paroissiales périodiques et relance le culte de la Sainte Vierge par un sermon intitulé a la grandeur de la Sainte Vierge

\footnotetext{
20 Paul Lesourd, op. cit., p. 201.

21 C.P., 27 septembre 1840.

22 C.P., 10 septembre 1843.

23 C.P., 13 septembre 1840.
}

24 Environ 5,000 hommes et 5,000 femmes assistaient quotidiennement aux sermons.

25 C.P., 13 septembre 1840. Il semble que l'intempérance soit une cause très importante de l'abandon des sacrements. 
et le droit qu'elle a à notre dévotion et à notre confiance ${ }^{26}$ ». Elle influence aussi directement la fréquentation des sacrements et surtout, précipite la fondation de la société de tempérance qui influencera les Québécois jusqu'à la fin du XIX ${ }^{\bullet}$ siècle et demeurera, pour les vingt années ultérieures, le point de concentration des efforts cléricaux dans le redressement des mours populaires.

Le 5 septembre 1841, un an après la retraite paroissiale, le curé Baillargeon annonce du haut de la chaire la première retraite générale du clergé. Il signifie en substance que c'est au tour des clercs de se sanctifier. Désormais jusqu'en 1860 , le clergé aura sa retraite quasi annuelle ${ }^{27}$. Quant à la retraite paroissiale, le curé s'efforce de la régulariser. Toutefois s'il ne réussit pas toujours, faute de prédicateur ou de temps favorable, il peut dans ces cas, suppléer à cette carence par la neuvaine du carême ${ }^{28}$.

Les deux années qui suivent la retraite de 1840 démontrent que la tempérance s'avère un moyen efficace de raffermir les convictions religieuses. Le curé ne manque pas de l'exploiter. Les trois retraites suivantes, celle de 1843 , prêchée par l'abbé Joseph-Fortunat Aubry, supérieur du séminaire de Québec, celle de 1847, par Armand-François de Charbonnel et celle de 1849, par le grand vicaire Alexis Mailloux, nous apparaissent essentiellement comme des campagnes de tempérance. Le curé Baillargeon, à l'occasion d'un prône dans lequel il loue le supérieur du séminaire, nous donne un aperçu du résultat de la tempérance:

Le concours a toujours été en augmentant, et dans les derniers jours, la foule qui se pressait aux sermons égalait celle que les sermons de l'évêque de Nancy avait attirée. Mais les instructions de monsieur Aubry étaient beaucoup mieux entendues et plus pratiques, et plus solides, et plus à la portée de son auditoire que celles de l'évêque de

26 Quoiqu'il soit difficile de juger de l'état réel du culte de la Sainte Vierge, certains indices permettent de situer autour de la prédication de Forbin-Janson, un renouveau dans ce domaine. Aucun sermon des neuvaines de la grâce, aussi appelées neuvaines du carême, ne traite de la Sainte Vierge. En général, les fêtes de l'Assomption et de l'Immaculée Conception sont les seules occasions de prêcher sur ce sujet. La retraite de Forbin-Janson innove donc en ce sens que pour la première fois, dans une récollection populaire, la dévotion mariale est particulièrement recommandée. $\mathrm{Et}$ davantage, après son sermon, l'évêque de Nancy consacre solennellement son auditoire à la Sainte Vierge. Dans ces circonstances, si la retraite de 1840 n'est pas l'occasion d'un renouveau en ce domaine, elle est certes le point de départ d'une prise de conscience, puisqu'en novembre 1840 , l'évêque incite le curé de Notre-Dame à faire du recrutement pour la confrérie de la Sainte-Famille; le 2 octobre 1842, le curé Baillargeon rétablit la confrérie du Saint-Rosaire et le 30 avril 1843 débutent les premières manifestations du mois de Marie à Québec.

27 Il n'y a pas eu de retraite du clergé en 1845, 1847, 1848, 1849 et 1854. Voir B.R.H., $\mathrm{n}^{\circ}$ 7, 1901 , p. 277.

28 Selon les Cahiers des Prônes, il y eut, de 1840 à 1860, huit retraites ou jubilés prêchés dans la paroisse Notre-Dame de Québec, soit en 1840, 1843, 1847, $1849,1851,1852,1854$ et 1859. 
Nancy. Les fruits aussi ont été plus abondants. Le nombre de ceux qui sont revenus à Dieu, dans la sincérité de leur cour, dans ces jours de retraite, a été extraordinaire. Mais la tempérance a été le moyen universel par lequel Dieu a ramené ces pécheurs. Presque tous ceux qui se sont convertis étaient rangés depuis un certain temps sous les enseignes de l'abstinence totale et n'attendaient plus que le moment pour se réconcilier. On s'est assuré que pas moins de 1,500 hommes se sont approchés de la communion... bon nombre se sont rendus dans les semaines qui l'ont suivie, attirés par l'exemple $29 .$.

Les retraites donnent ainsi au curé l'occasion de multiplier les manifestations religieuses. Il suffit de voir le taux de fréquentation des sacrements, surtout de la confession et de la communion, après ces prédications, pour comprendre l'influence qu'elles ont sur le peuple. La moyenne des confessions pascales chez les Irlandais et Canadiens français de la paroisse atteignait environ 6,500 depuis 1833. Après la retraite de Forbin-Janson, en 1841, elles s'élèvent au nombre de 8,432. Laissons le curé commenter lui-même ces chiffres:

Au sujet du nombre des confessions (...), il faut savoir que la population de la paroisse n'avait point été augmentée; que le nombre des confessions durant le carême des années précédentes ne s'élevait guère au-delà de 7,00030 . - C'est donc près de 1,500 confessions et communions de plus cette année que les années précédentes. - Mouvement religieux, augmentation de piété et de foi remarquable dû à la retraite de l'automne précédent, et surtout à l'établissement de la Société de Tempérance ${ }^{31}$.

\section{Il écrit en avril 1843:}

Nous vous annoncions, il y a deux ans que le nombre des Pâques avait considérablement augmenté. Ce nombre s'est soutenu l'année dernière; et nous avons la consolation de vous annoncer qu'il s'est encore augmenté cette année. Les causes: les retraites et la tempérance $\mathbf{3 2}$.

Il n'y a pas d'équivoques, le curé a les preuves de l'efficacité religieuse des retraites. Il reconnaît aussi que le succès des retraites est lié à l'existence de la société de tempérance. Mais faut-il que l'intempérance soit un problème si grave pour qu'il suscite une telle prise de conscience? Nous le croyons; les 160 hôtels et tavernes et les 18 marchands de vins et liqueurs dénombrés en 1857 sont bien éloquents sur ce sujet ${ }^{33}$. L'usage des boissons alcoolisées est si bien ancré dans les mœurs qu'il faut plus que des prédications et une société de tempérance pour

$29 \quad$ C.P., 5 février 1843.

30 Les statistiques du Cahier des Prônes de 1832-1843 nous indiquent que le nombre de 6,830 confessions, en 1833 , est le plus élevé.

31 C.P., 18 août 184.1.

32 C.P., 30 avril 1843.

33 E. C. GuIllet, Pioneer Inns and Taverns, t. II, Toronto, Ontario Publishing Co., p. 49. L'auteur reproduit la liste des tavernes de Québec telle qu'inscrite dans Canada Directory of $1857-58$ de Lovell. 
révolutionner la coutume. D'ailleurs le découragement en face de la situation économique pénible et les exigences de nos hivers rigoureux ont des conséquences qu'il ne faut pas ignorer sur les comportements face à l'alcool. C'est sans doute ce qui explique l'échec de la retraite de 1847, où le curé, après avoir exhorté ses fidèles à la piété en brandissant le spectre du choléra qui ravage l'Irlande, revient à la charge: « On a fortement exhorté à la tempérance durant la retraite... Peu ont répondu... Serait-ce que cet avertissement n'aurait pas été compris, ou bien parce qu'on ne veut pas ${ }^{34}$ ?

Voilà une limite à l'influence du curé. Mais les moyens d'action à sa disposition sont nombreux: outre son engagement dans l'éducation, le bien-être social et le redressement des mœurs, formes d'engagement que nous avons volontairement ignorées ici ${ }^{35}$, d'autres manifestations de son rôle sur le plan strictement religieux ${ }^{36}$, concourent à accroître son influence. Ce sont les indulgences, les processions, les messes et les offices.

\section{Les indulgences}

Les indulgences ont toujours été, durant cette partie du XIX' siècle, un moyen efficace de stimuler la ferveur religieuse. Il n'y a pas un office, une cérémonie ou une "bonne œuvre" quelconque, dont on désire le succès, qui ne soient accompagnés d'indulgences partielles ou plénières. Le paroissien y attache une importance particulière; il en fait une de ses motivations essentielles. Quand en 1845, l'évêque suspend la cérémonie des Quarante Heures, il renvoie l'indulgence plénière qui y était attachée au dimanche de la Pentecôte ${ }^{37}$. Ces indulgences assurent presque toujours la participation aux entreprises cléricales et l'évêque s'en sért pour promouvoir ses œuvres de charité: lors des différents jubilés ou triduums, les indulgences accordées sont liées à certaines obligations comme la confession, la communion et la participation à

34 C.P., 6 juin 1847. Il arrive très souvent que le curé reproche aux fidèles de ne pas tenir les engagements pris lors des retraites.

${ }_{35} \mathrm{~A}$ ce sujet, consulter notre thèse, Aperçu du rôle social et religieux $d u$ curé de Notre-Dame de Québec, 1840-1860. Thèse présentée à l'Ecole des Gradués de l'Université Laval en mai 1968.

36 La brève description que nous venons de faire des retraites à Notre-Dame de Québec ne se veut pas une analyse définitive de la prédication dans cette paroisse. Nous ne connaissons à peu près rien des sermons du dimanche et des fêtes d'obligation. Des neuvaines du carême, nous ne connaissons que les titres des sermons. Voici les sujets des sermons qui reviennent le plus souvent lors des neuvaines entre 1840 et 1860 : le symbole, la mort, la prière, l'enfer, la miséricorde, l'amour de Dieu, le ciel, la persévérance, les mauvais livres, les danses.

37 C.P., 4 mai 1845. L'office des Quarante Heures est suspendu momentanément avec la suppression de l'office des secondes et des troisièmes fêtes de Noël, de Pâques et de la Pentecôte. Par contre, même s'il est supprimé et ne s'annonce plus officiellement, certaines congrégations religieuses en conservent la dévotion. Il semble que ce soit le peu d'assiduité des fidèles qui ait occasionné cette suppression. 
deux œuvres de charité; or il arrive qu'il dicte lui-même les œuvres de charité nécessaires pour remplir les conditions. C'est le cas du jubilé de 1852, où les indulgences sont accordées à ceux qui souscrivent de leur argent à l'œuvre de la propagation de la foi et à la construction, à Québec, de l'édifice des Sceurs de la Charité.

Le curé, promoteur de cette dévotion, en connaît la puissance et fait du mieux qu'il peut pour l'activer. Il annonce aux prônes du 24 décembre 1843: a Noụs prinns les personnnes qui ne sont point disposées à communier de ne point se présenter au confessional cet après-midi, ni demain matin, afin de ne point prendre le temps de celles qui désirent gagner les indulgences. "Mais il nous semble que l'importance qu'on accorde aux indulgences vaut surtout sur le plan psychologique pour des chrétiens qui sentent le besoin d'accumuler les grâces qu'ils se méritent, à une époque où les malheurs si nombreux, s'interprètent en terme de " châtiment ». Harcelés par la crainte du péché, annonciateur de nouveaux marasmes, n'est-il pas normal qu'ils concrétisent par l'accumulation d'indulgences, les résultats des prières qui pourraient calmer la colère de Dieu ${ }^{38}$. Cette situation que nous pourrions être tenté de qualifier d'exploitation par le clergé de la crédulité populaire, ne peut s'interpréter de cette façon. C'est bien plus un phénomène de mentalité autant cléricale que populaire, résultant de la « croyance aveugle " en l'intervention constante de la «divine providence " sur la destinée des peuples. En fait, les résultats servent aussi bien le clergé que le peuple: tout en assurant une participation satisfaisante à tous les moyens mis en ouvre par le clergé, les indulgences réconfortent la conscience du peuple.

\section{Les processions}

Les paroissiens honorent particulièrement de processions la fête de saint Marc, les Rogations et la Fête-Dieu. Cette dernière est la plus prestigieuse et la plus importante des trois, car le saint sacrement est porté par le curé à travers les rues principales de la ville pour être déposé sur un reposoir orné de fleurs, de verdures et des plus riches

38 Nous avons déjà noté que le curé attribue en partie à la débauche et au péché les marasmes qui accablent Québec depuis le choléra de 1832. Le curé se sert aussi de cette explication comme moyen de prévention : après les deux plus graves incendies du siècle, à Québec, en 1845, le curé sermonne vertement les « jeunes libertins * qui en septembre 1846, se tiennent à la porte de la chapelle des Ursulines et insultent les jeunes filles. A cette occasion, l'archevêque autorise les Ursulines, incapables de maintenir l'ordre, à fermer momentanément les portes de la chapelle. Le curé s'exprime ainsi au prône du 20 septembre 1846 : CPauvre ville de Québec... tes enfants, tes rrais enfants, les enfants de l'Eglise te préparent de nouveaux malheurs!' Ils oublient Dieu. Ils outragent leur Père et leur unique protecteur... Et il n'y a point de paix, point de prospérité, point de bonheur pour les impies. Dieu de miséricorde ayez pitié de nous... ne nous châtiez point selon la multitude de nos iniquités.... 
candélabres. On ne ménage pas les effets; tout l'effectif religieux de la paroisse se met en branle pour donner à cette fête le plus de prestige et de piété possible. Les citoyens sont avertis quinze jours à l'avance des rues empruntées pour la procession. Riches et pauvres sont invités à décorer leurs demeures. Le curé supervise et encourage, soit qu'il félicite les habitants de telle ou telle rue pour " avoir mis tant de zèle à la décoration ", soit qu'il incite à faire davantage: "Pour tout le moins, dit-il, les citoyens doivent se faire un devoir d'orner leur maison de verdure. C'est une dépense assez légère pour chacun, mais qui deviendrait lourde pour la fabrique. Celle-ci est déjà chargée d'orner les demeures de nos concitoyens des autres croyances ${ }^{39} \ldots$ En effet la fabrique veille à ce que chaque demeure soit décorée; quand une famille d'un culte différent n'a pas les moyens ou ne veut pas coopérer, elle procède, à ses propres frais, à la décoration. Même les écoliers collaborent à la fabrication des fleurs artificielles et participent au " chœur d'anges ".

L'ordre de la procession et le protocole sont dictés du haut de la chaire le dimanche précédant la cérémonie. Les personnes concernées se réunissent et étudient à l'avance chacun de leurs mouvements. Le curé nous le décrit:

Pour le bon ordre de la procession chacun de ceux qui doivent en faire partie doit connaître la place et le rang qu'il doit occuper. Les dames des confréries de la Sainte-Famille, du Scapulaire et de la bonne Sainte-Anne doivent suivre la bannière sur deux rangs, observant de se suivre toutes à la distance d'environ deux petits pas. Les confrères de Sainte-Anne sont priés de suivre aussi la bannière, et de former en dehors des dames, deux lignes, une de chaque côté de la rue, pour les protéger et les garder contre les curieux qui se pressent souvent sur le passage de la procession. Messieurs les congréganistes sont invités à former de chaque côté du clergé, une ligne qui doit commencer à la suite des confrères de Sainte-Anne et se terminer un peu en avant du Dais, pour laisser la place aux marguillers qui doivent prendre la place à côté du Dais et prolonger la ligne à une petite distance en avant. Les congréganistes et les confrères en formant cette ligne pourront protéger la procession de la bannière jusqu'au Dais. Ils sont priés de ne laisser aucun vide $40 .$.

Et ajoute-t-il, chacun doit manifester « la sincérité de sa foi à l'extérieur " par la piété.

Alors que tant d'éclat, tant de zèle et de dévouement pourraient nous laisser croire à un déploiement maximum des ressources de la cure, nous sommes littéralement ébahis par le lustre des processions de

39 C.P., 27 mai 1869. D'autres écrits du curé, entre les années 1850-1860, disent en substance la même chose.

40 C.P., 29 mai 1842. 
la décennie suivante. Avec l'arrivée à Québec de l'éclairage au gaz, les processions se font le soir, et sur recommandation du curé, toutes les maisons sunt illuminées ${ }^{41}$. Les élèves des différentes écoles y participent. En 1866, la milice volontaire offre ses services pour faire garde d'honneur; une salve de canons annonce la mise en marche. "Rien de semblable, dira le curé, n'a été vu depuis le régime français dans notre pays ${ }^{42}$." Puis c'est au tour du gouverneur, des juges, des dignitaires de l'université et des avocats à y être invités. Nous voilà en plein cœur de la moniée uitramoniaine. Désormais, rien ne sera trop grand pour l'Église. Le curé ira même jusqu'à inviter les propriétaires de commerce à fermer leurs portes dans la matinée de la fête : "Ne nous laissons pas vaincre en générosité, dira-t-il, par les croyances séparées qui savent bien faire ce sacrifice à la moindre fête qui les intéresse ${ }^{43}$. * Hélas ! ces énormes préparatifs, cette démonstration éloquente de piété et de ferveur s'évanouissent, au témoignage même du curé, devant une température maussade ou des rues boueuses ${ }^{44}$.

\section{Remarques sur l'assistance à la messe et aux offices}

Ces quelques témoignages révélateurs d'un accroissement de la piété populaire n'en recouvrent pas tous les aspects. D'autres attitudes que dévoilent les Cahiers des Prônes montrent la persistance d'habitudes traditionnelles appelées à une longue survivance. Les incitations répétées du curé de ne pas quitter la messe et les offices avant la fin et de ne pas sortir à l'heure du sermon, les remarques sur la profanation du dimanche et sur le peu d'assiduité aux offices, les défenses aussi nombreuses de se rassembler devant l'église ou dans les sacristies et les escaliers pour y entendre la messe; voilà des remarques désagréables qui décrivent bien le non-conformisme de certains. Que dire des avertissements aux fidèles des arcades et des jubés de ne rien jeter dans la nef, ni de cracher ${ }^{45}$ ? Que penser du fait qu'on ait supprimé la messe de minuit à cause des désordres ${ }^{46}$ ? Du fait qu'on ait aboli les vêpres et la cérémonie des Quarante Heures faute de fidèles? Que penser aussi des avertissements aux femmes qui paradent dans les allées centrales après le début de la messe? Des paroissiens qui envoient leurs enfants chercher un plein seau d'eau bénite du samedi saint ${ }^{47}$ ? Autant d'anec-

41 Le curé écrit dans ses prônes, le 18 juin 1871 : . La procession aura lieu mercredi soir à 9 heures pour que l'illumination produise son effet. ,

$42 \quad$ C.P., 27 mai 1866.

$43 \quad$ C.P., le 18 juin 1871.

44 C.P., 21 juin 1840,10 juin 1855.

$45 \quad$ C.P., 4 avril 1852.

46 C.P., 25 décembre 1860. La messe de minuit est rétablie en 1860 avec l'arrivée de l'éclairage au gaz. Cependant le curé se dit toujours prêt à fermer l'église s'il y a de nouveaux désordres.

47 C.P., 4 avril 1852. 
dotes qui illustrent certains aspects de l'esprit religieux d'une partie des paroissiens ${ }^{48}$.

En conclusion de cette première partie, rappelons que la pauvreté de la documentation consultée ne nous a pas permis d'établir les comparaisons nécessaires pour évaluer rigoureusement l'augmentation de la ferveur religieuse. Par contre, quelques données statistiques sur la fréquentation de la communion pascale avant et après 1840, l'apparition des retraites et des campagnes de tempérance, la fondation d'organismes religieux, l'engouement pour les indulgences autant que l'empressement des paroissiens à participer à la procession de la Fête-Dieu semblent indiquer avec certitude l'existence d'un renouveau religieux à la paroisse Notre-Dame de Québec. Toutefois, certaines remarques désagréables, de la part du curé, sur la piété des fidèles, nous invitent à nous interroger sur la profondeur des sentiments religieux manifestés.

Les explications de la pratique religieuse sont toujours complexes. A ce sujet, Gabriel Le Bras écrivait: "Discerner exactement la part de la foi, de la coutume, de la contrainte dans les actes religieux, est audessus des forces de l'homme. "Et loin de conclure à l'inutilité de telles recherches, l'auteur poursuivait: "Mais combien de données nous éclairent! Le milieu, son histoire et son état présent, voilà ce qu'il nous faut avant tout connaître ${ }^{49}$." Par exemple, quelle est la part de la foi, de la contrainte sociale et de l'éloquence des prédicateurs, dans le succès des campagnes de tempérance? Pourrions-nous y répondre que nous aurions cerné les mobiles les plus intimes de l'homme, ceux qui justifient ses relations avec la divinité. Certes, le but visé semble impossible à atteindre. Mais ces interrogations ne permettent-elles pas d'émettre certaines hypothèses intéressantes pour la compréhension de notre passé religieux et pour l'explication de situations présentes?

Nous avons déjà esquissé des réponses à ces questions, dans la première partie, quand nous avons décrit la mise en scène macabre et spectaculaire qui accompagne les prédications et quand nous avons supposé que l'engouement pour les indulgences pouvait provenir d'un désir de se prémunir contre la colère d'un Dieu vengeur. Un autre exemple: celui

48 Cette étude ne nous permet pas d'évaluer le nombre de ces paroissiens. Il est cependant permis de croire qu'il est assez élevé. Il suffit pour s'en rendre compte, de consulter le taux de fréquentation à la communion pascale : de 6,500 avant 1840 , le nombre est passé à 8,432 après la retraite de Forbin-Janson. Or même ce chiffre qui à première vue paraît élevé n'est pas considérable sur une population d'environ 16,500 catholiques.

49 Gabriel LE Bras, Etudes de sociologie religieuse, Paris, P.U.F., 1955, T. I, p. 223. 
des offrandes de messes recommandées ${ }^{50}$ que nous avons dénombrées dans les Cahiers des Prônes des années 1844, 1845 et 1849. Ces années ont été choisies en fonction de la situation économique et sociale. Álors qu'en 1844, les citoyens de Québec jouissent d'une relative prospérité, seulement 50 messes recommandées sont inscrites dans les cahiers. En 1845 , ce nombre augmente à $73 ; 35$ de ces messes sont offertes durant les mois de juin et de juillet, alors que deux incendies ( 25 mai et 28 juin) détruisent les deux tiers de la ville. En 1849, un choléra, commencé le 5 juillet, fait 1,066 victimes dans ìa vilile de Q̂uëbec. L̉es 110 messes offertes s'échelonnent autour des périodes où les dangers de mortalité sont les plus imminents. (Voir le tableau graphique en page 95.)

Dans ce cas bien précis, la crainte d'un Dieu vengeur semble être la motivation principale de la ferveur des paroissiens - et sur ce point le tableau graphique est éloquent. N'en serait-il pas ainsi de l'ensemble des manifestations du réveil religieux de 1840 ?

Les historiens qui se sont penchés sur le problème de l'interprétation de ce mouvement ont apporté quelques explications qui nous semblent nettement insuffisantes. Qu'ils l'attribuent à l'uniformité de pensée cléricale après l'érection du diocèse de Montréal en 1836, ou encore au prestige, à la renommée et aux qualités personnelles de $M^{\mathrm{gr}}$ de Forbin-Janson ${ }^{51}$, nous ne pouvons accepter cette interprétation comme satisfaisante, même si elle est à considérer. De telles recherches ne pourront nous satisfaire que lorsqu'elles seront orientées vers la connaissance des causes assez puissantes pour expliquer des comportements collectifs multiples, ou mieux, lorsqu'elles seront orientées vers la connaissance du «milieu, de l'histoire et du présent " des Québécois de la première moitié du $\mathrm{XIX}^{\bullet}$ siècle. C'est à partir de cette voie de recherche, déjà tracée par Gabriel Le Bras, que nous faisons ici une esquisse de la situation socio-économique et politique ${ }^{\mathbf{5 2}}$.

Depuis 1826, la misère et souvent la famine s'installent graduellement dans les campagnes, à la suite de plusieurs mauvaises récoltes.

50 Ce sont des messes chantées. Seules ces messes sont annoncées et inscrites dans les Cahiers des Prônes. Il y a possibilité d'en célébrer une par jour. Notons que la majorité des messes dénombrées ici ont été offertes en a action de grâces, ou " pour être préservé des maladies *.

51 Pour avoir une juste idée de l'enthousiasme suscité par les retraites de Mgr de Forbin-Janson, à travers le Bas-Canada, consulter Léon Poulior, La réaction catholique de Montréal, 1840-1841, Montréal, Imprimerie du Messager, 1942, 119 p. F.-X. Côté, Mgr de Forbin-Janson, évêque de Nancy et de Toul, et le mouvement religieux du Québec vers 1840 , S.C.H.E.C., 1941-1942, pp. 95-118.

52 Cette esquisse de la situation socio-économique et politique doit être complétée par une étude de la structure de la société. Pour les fins d'une analyse complète, il faudrait nécessairement étudier les perceptions propres à charun des groupes. Ainsi ne serait-il pas utile de savoir comment l'élite a été touchée par la conjoncture socio-économique, la politique et aussi le réveil religieux de 1840 ? 
TABLEAU GRAPHIQUE DES OFFRANDES DE MESSES RECOMMANDÉES EN 1844, 1845 ET 1849.

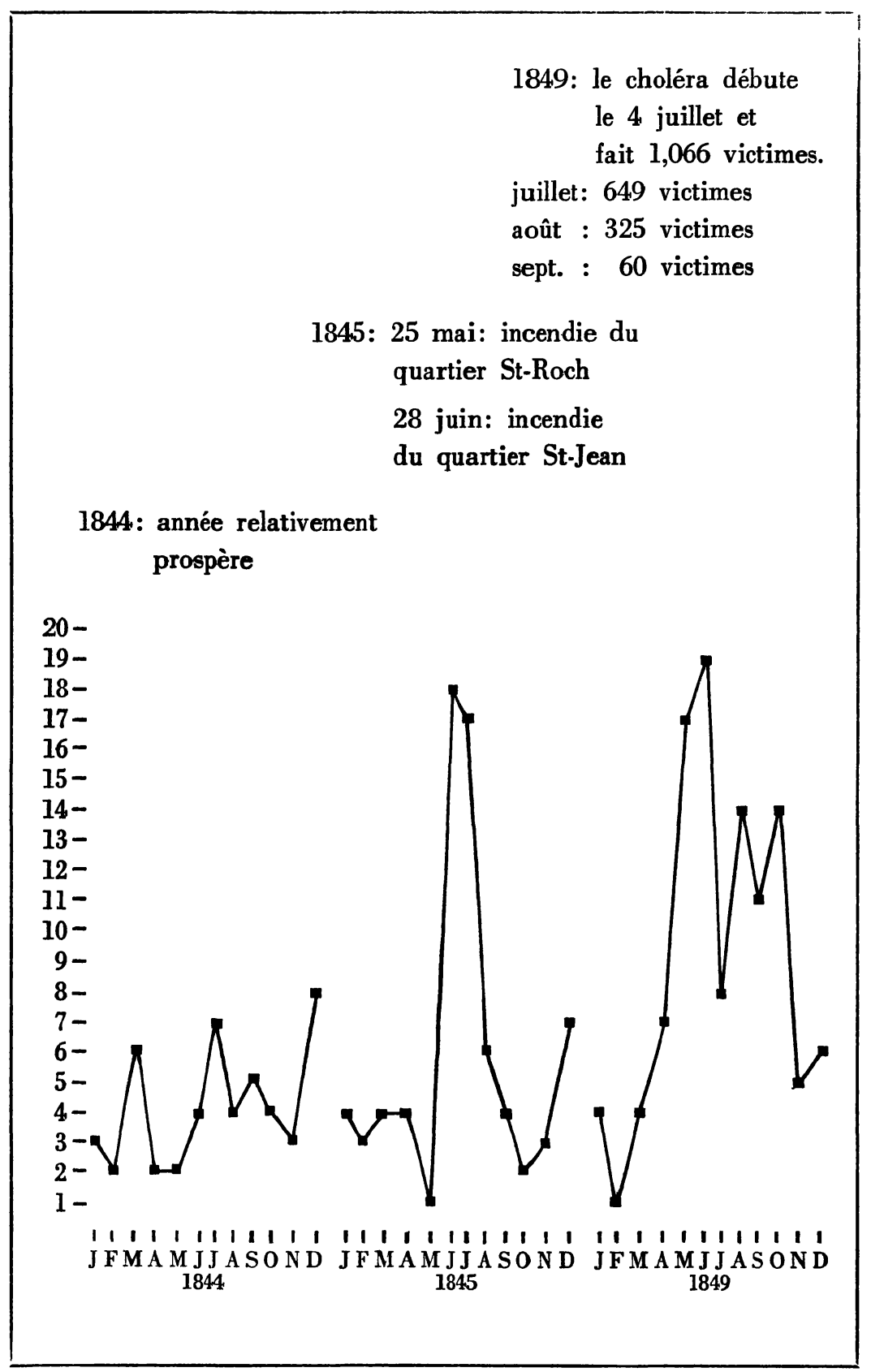


Le malaise se répercute sur les villes et s'accentue suivant les déboires du commerce et de l'industrie intimement liés à la conjoncture internationale. La situation est telle que la famine ou du moins les privations imposées par la disette et la pauvreté surgissent presque à chaque hiver chez les Canadiens français peu fortunés ${ }^{53}$. Mais le peuple n'a pas encore vu la fin de ses malheurs, car deux terribles épidémies de choléra, en 1832 et 1834, s'ajoutent aux misères coutumières pour semer la mort de paroisse en paroisse. Ajoutons-y les incidents locaux ${ }^{54}$ (incendies, Hléaux et autres malheurs) qui aggravent a l'echeile paroissiale la situation sociale générale, et nous comprendrons l'importance de ces incidents sur la motivation psychologique à la Rébellion.

D'un autre côté, l'échec de l'insurrection contrecarre pour un temps les aspirations politiques, économiques et nationalistes des Canadiens français. La répression sanglante de Colborne n'est pas encore oubliée que le parlement impérial impose l'acte d'Union, interprété comme le désir évident d'angliciser ce peuple rebelle.

Voilà résumée la série d'épreuves qui, en près de quinze ans, s'est abattue sur les Canadiens français. Sur tous les plans, politique, économique et social, ils ont subi des échecs; et le peuple en est d'autant plus conscient que le clergé n'a cessé de les souligner. A chaque épreuve, le curé lui rappelle ses devoirs religieux qu'il a mal accomplis et voit dans ce manque de piété la cause des malheurs. Il n'y a pas un des faits énumérés ci-haut - du moins dans la paroisse Notre-Dame - qui n'aient été interprétés comme une vengeance divine ou une punition de l'impiété populaire ${ }^{55}$.

Ainsi nous semble-t-il que toutes ces raisons poussent les Canadiens français à se replier sur eux-mêmes et les disposent à entendre la voix du clergé dont ils avaient négligé les appels au moment des insurrections. Ils sont psychologiquement prêts à se jeter désespérément sur la seule solution présentée, la religion. C'est un exutoire mais aussi l'explication qu'ils trouvent à leurs maux. Ainsi quand Mgr de Forbin-Janson arrive dans les paroisses du Bas-Canada, les foules se précipitent à sa rencontre et manifestent une piété et une ferveur jamais vues auparavant.

Parallèlement à ce climat psychologique provoqué par la situation politique, économique et sociale, d'autres événements contribuent à

53 Sur ce sujet, consulter Fernand Ouelnet, Histoire économique et sociale du Québec, 1760-1850, structures et conjoncture, Montréal, Fides, 1966, pp. 405-537. Cinsulter aussi notre thèse, Aperçu du rôle social et religieux du curé de NotreDame de Québec, 1840-1860.

:i4 A Québec, nous pouvons ajouter l'incendie de la Basse-Ville, en 1836.

35 Cette justification a posteriori des événements ne nous paraît pas propre au seul clergé canadien-français. En France, Forbin-Janson et ses missionnaires en ont abusé. 
l'accroissement de la ferveur religieuse : c'est l'évolution numérique du clergé, sa réorganisation et partant la diversité de ses influences. Nous avons déjà traité de ce sujet dans la première partie; répétons ici que le nombre des prêtres a doublé entre $1830(225)$ et 1840 (464), et quadruplé entre 1830 et 1860 (948). Les communautés religieuses se sont multipliées. Mais le point saillant de la réorganisation ecclésiastique n'est-il pas l'érection du diocèse de Montréal en 1836 ? A ce sujet, le chanoine Groulx écrit :

Les luttes pénibles faites par quelques Sulpiciens à Mgr Lartigue et aux archevêques de Québec, au sujet de la nomination du premier suffragant de Québec, à Montréal, de sa juridiction, du lieu de sa résidence, du choix de sa cathédrale, des honneurs à lui rendre, de la cure de Montréal, de la reconnaissance par Rome du coadjuteur de Mgr Signay, de l'agrégation à la compagnie sulpicienne, de sujets canadiens et de sujets de France, tous ces différends et quelques autres plus souvent graves que légers, devaient fatalement semer l'insubordination. Des partis se sont formés parmi les prêtres et parmi les laïcs. Sur la question du suffragant, on ferraille à coups de brochures entre clercs, entre laïcs et clercs. La querelle est portée dans les journaux ${ }^{56}$ et jusque dans la chaire (...). On soutient que la * prétendue érection du District Épiscopal, est contraire aux lois canoniques, à l'ordre établi légalement dans le diocèse (...) Cet esprit de révolte ne gagne pas heureusement la majorité du clergé de Montréal, ni même tout le Séminaire. Mais trop longtemps entretenu, il aura des bouffées volcaniques jusqu'en $1836 \mathbf{5 7}$.

Ainsi l'érection du diocèse de Montréal (1836) si elle ne met pas complètement fin aux luttes gallicanes ${ }^{58}$ qui, à un moment donné, ont presque divisé l'Église du Québec en deux factions et laissé le peuple en face de nombreux exemples d'insubordination, amenuise les querelles ${ }^{59}$ et les place à l'abri de la critique populaire.

L'éducation, autre élément de l'influence cléricale, fait d'énormes progrès en ces années. Du côté de l'enseignement supérieur, quatre nouvelles fondations, le Séminaire Sainte-Thérèse en 1825, de Chambly la même année, de Sainte-Anne-de-la-Pocatière en 1829 et de l'Assomption en 1832 s'ajoutent aux anciennes institutions, Québec (1663), Montréal

56 Lionel Groulx, a La situation religieuse au Canada français... , op. cit., p. 54, note 19, l'auteur écrit : . Voir en particulier Le Spectateur Canadien, 24 et 28 juin 1823. Voir sur la part de cette polémique dans les journaux, entre autres choses, l'aveu de l'abbé ChaBoIllez, dans sa Réponse de Messire Chaboillez... (Montréal, 1824, T. A. TURNER), p. 55. R.A.P.Q., 1936-37, pp. 218, 232-234, 237-238, 242, 251. Pour les autres querelles, voir, en particulier l'analyse de la correspondance des évêques de Québec dans R.A.P.Q., 1934-1935, 1935-1936, 1936-1937.,

57 Ibid., pp. 54-55.

58 Nous employons ici ce terme à l'instar de nombreux auteurs. Cependant, nous nous interrogeons sur le sens véritable de ces luttes. Ne sont-elles peut-être que des querelles de préséance et des conflits de personnalités ?

69 Gaston CARRIÈre, * L'Eglise canadienne vers 1841 ,, Revue de l'Université d'Ottawa, 1954, p. 83. 
(1767), Nicolet (1803) et Saint-Hyacinthe (1809) pour former le cadre du recrutement ecclésiastique ${ }^{60}$. Mais c'est surtout du côté de l'enseignement primaire que le clergé dirige ses efforts. En plus d'encourager les citoyens à envoyer assidûment leurs enfants à l'école, il se charge personnellement de l'enseignement du catéchisme et parfois contrôle indirectement l'engagement des professeurs en imposant ses conditions ${ }^{61}$. Aussi fonde-t-il et finance-t-il ses propres écoles, car, bien entendu, l'enseignement religieux demeure sa première préoccupation. L'arrivée à

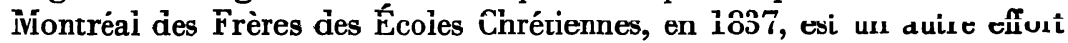
en ce sens.

Bref, alors que les événements de la décennie 1830-1840 portent le peuple canadien-français à se tourner vers la religion et le clergé, ce dernier s'organise et accentue son influence. L'arrivée de Mgr de ForbinJanson canalise définitivement les aspirations populaires et encourage le clergé à faire davantage. La simple énumération des ouvres cléricales après 1840 nécessiterait de nombreuses pages; écartons-nous-en ici, car notre seule ambition était d'émettre une hypothèse d'explication du réveil religieux de 1840 .

\section{CONCLUSION}

La valeur hypothétique de ces considérations sur le conditionnement psychologique et social de la pratique religieuse dans la paroisse Notre-Dame et aussi dans l'ensemble du Bas-Canada rend d'autant plus difficile un jugement sur la profondeur des sentiments religieux observés que toutes ces manifestations, au-delà de leurs conditionnements, supposent l'existence de la foi, phénomène quasi insondable. Qui niera que la ferveur religieuse est le produit de la foi ? Et la crainte de Dieu que nous avons décrite comme la motivation principale des comportements religieux n'est-elle pas une forme de la foi ? Son influence ne pose donc pas de problèmes; là où nous sommes en droit de nous interroger, c'est sur les qualités de cette foi, sur son contenu, sa pureté, sa vigueur. Pour le moment, nos études ne nous permettent pas de répondre à ces questions. Toutefois les hypothèses avancées semblent indiquer que les moyens mis en euvre par le clergé pour ranimer la pratique religieuse des Québécois de 1840, font davantage appel à l'émotivité qu'à la raison, de sorte qu'il serait plausible que la pratique religieuse régresse, sitôt cette contrainte socio-psychologique évanouie.

René HARDY,
professeur d'histoire, Québec.

$60 \quad$ Ibid., p. 79.

61 A la paroisse Notre-Dame, il arrive souvent que le curé défende aux parents d'envoyer leurs enfants aux écoles de professeurs laïques qui acceptent les classes mixtes (garçons et filles). De plus, les noms des professeurs laïques acceptés par le curé sont annoncés du haut de la chaire. 